\title{
Des territoires à la territorialisation
}

From Territories to Territorialization

Isabelle Pailliart

\section{(2) OpenEdition}

\section{Journals}

Édition électronique

URL : http://journals.openedition.org/edc/7635

DOI : 10.4000/edc.7635

ISSN : 2101-0366

Éditeur

Université de Lille

\section{Édition imprimée}

Date de publication : 1 juin 2018

Pagination : 147-160

ISBN : 978-2-917562-19-2

ISSN : $1270-6841$

Référence électronique

Isabelle Pailliart, « Des territoires à la territorialisation », Études de communication [En ligne], 50 | 2018, mis en ligne le 01 juin 2020, consulté le 03 janvier 2020. URL : http://journals.openedition.org/edc/

7635 ; DOI : 10.4000/edc.7635 


\section{Des territoires à la territorialisation \\ From Territories to Territorialization}

Isabelle Pailliart

Université Grenoble Alpes, laboratoire GRESEC

isabelle.pailliart@univ-grenoble-alpes.fr 
À partir de l'analyse des médias et des supports d'information et de communication, trois conceptions du territoire se dégagent principalement. Elles mettent en évidence le fait que le territoire est construit et que cette construction représente un enjeu pour les différents acteurs. Les médias locaux prennent part à ces conceptions territoriales, tout comme la production d'informations dans le cadre d'opérations de participation ou lors de débats et de conflits locaux. L'article propose alors de considérer le territoire comme un condensé de tensions et d'affrontements entre différents modèles de développement social et économique.

Mots-clés : communication, médias, espace public, territoires, associations.
Media and information analysis reveals three main conceptions of territory. These conceptions highlight the fact that territories are constructions and that different actors have a stake in the process of such construction. Local media take an active part in conceptions of territory, as does information produced through participatory action or local debate and conflict. This article thus considers territory as a condensed reflection of the tensions and confrontations among different models of social and economic development.

Keywords: communication, media, public space, territories, associations. 
En plus d'une vingtaine d'années (1995-2017), la revue Études de communication (EDC) a abordé les rapports entre les territoires et les médias, à l'occasion de six dossiers et d'une trentaine d'articles. Les intitulés de dossiers indiquent une cohérence certaine (ce qui est l'objectif éditorial d'un dossier) même si leurs dénominations varient : il s'agit par exemple de "l'espace régional » ( $\left.n^{\circ} 17\right)$, des «territoires » $\left(n^{\circ} 26\right)$ ou des « espaces urbains » $\left(n^{\circ} 31\right)$. Les différences renvoient bien à une tendance générale des analyses portant sur les territoires, du point de vue des sciences de l'information et de la communication pour lesquelles l'expression « territoires » renvoie à une catégorie générique au sein de laquelle se retrouvent toutes les approches qui abordent la spatialisation des activités d'information et de communication.

Ce positionnement trouve son explication dans l'histoire même des sciences de l'information et de la communication. En effet, très tôt la discipline, sur la question locale, s'est présentée en opposition à un modèle d'analyse "stato-centrée ". Le modèle "centre-périphérie a fourni une référence dominante, centrée sur les rapports entre l'État et les pouvoirs locaux, les élus locaux apparaissant comme des « relais privilégiés des organes territoriaux de l'État » (Gremion, 1976, 166). Cette approche présente dans la science politique est, alors, centrée sur une analyse du gouvernement local à partir de ses relations avec l'Etat. Ainsi « le système de gouvernement local est plus ou moins déterminé par les circuits de communication et les mécanismes d'échanges qu'il entretient avec l'État central - il est un gouvernement périphérique » (Mabileau, 1985, 556). Les sciences de l'information et de la communication ont développé une autre approche. Dans les années 70, leur institutionnalisation universitaire (Meyriat, Miège, 2002) correspond à une période d'exigence de démocratie locale et de revendications pour les formes localisées d'information et de communication : il s'agit de la "contre-information ", de l'information municipale, des premières expériences de télévision locale, puis au début des années 80 , des radios locales, des réseaux câblés et des services télématiques. La montée en puissance des collectivités territoriales a contribué au renforcement d'une autonomisation des informations locales. Il est souvent indiqué que les lois de décentralisation, menées par l'État, à partir de 1982, ont joué un rôle déterminant dans la montée en puissance de communication territoriale. Certes cette date sert de référence, mais elle ne suffit pas à expliquer un mouvement plus important. En effet, la communication des collectivités territoriales mais aussi la place des médias locaux et localisés se comprennent à la lumière de transformations plus profondes : l'évolution des rapports des individus avec les territoires, la construction progressive d'une gouvernance locale et les mutations du cadre étatique.

Les travaux en sciences de l'information et de la communication ont pris en compte ces transformations dès le début des années 80 . C'est par exemple en 1982, lors du troisième congrès de la Société française des sciences de l'information et de la communication que sont abordés les thèmes du pouvoir local, du rôle de l'État, de la place des associations, le plus souvent à travers 
ce qui est présenté comme des innovations techniques ou médiatiques. C'est, en effet, un double mouvement qui attire l'attention des chercheurs : la décentralisation (Kada et al., 2017) et la localisation de nouvelles " techniques d'information et de communication " proches chronologiquement et tendant à se renforcer mutuellement. La curiosité des chercheurs pour ces deux nouveautés, mêlée à l'intérêt des opérateurs qui s'est concrétisée par plusieurs contrats de recherche (Moeglin, 1991), a ainsi fait émerger un nouveau champ de recherche, d'autant plus riche qu'il introduisait des acteurs jusque-là peu visibles : les collectivités territoriales, les opérateurs publics ou privés et le tissu local de manière générale. Ainsi la recherche s'est-elle trouvée incitée à analyser les expérimentations et la "localisation " des stratégies des acteurs, que ceux-ci soient nationaux ou locaux. Les années 80 et les années 90 ont apporté des questionnements portant sur les rapports entre ces nouvelles techniques et la spatialité. Si certains travaux d'essayistes ont pensé l'espace dans un rapport conflictuel avec les techniques se traduisant par une disparition de celui-ci, si d'autres ont vu le territoire comme un simple support permettant le déploiement de stratégies nationales, si enfin quelques travaux ont considéré le local comme un élément permettant l'articulation entre l'offre technique et la « demande » sociale, plusieurs recherches dont un certain nombre ont été publiées dans la revue Études de communication ont pris le contre-pied de ces considérations. De manière générale, elles s'attachent à étudier la complexité des rapports sociaux au sein d'espaces différenciés pour engager une réflexion sur les processus de territorialisation et la place des médias au sein de ceux-ci. II s'agit bien d'analyser l'ensemble des dynamiques info-communicationnelles qui œuvrent à la construction des processus socio-territoriaux et qui se retrouvent, dans cet article, dans trois parties portant sur les conceptions territoriales, les médias et l'espace public. Ces trois entrées sont à associer étroitement pour rendre compte de la complexité de la territorialisation.

1.

\section{Espace ou territoire? Des approches différentes}

Les dossiers de la revue utilisent diverses dénominations : local, région, espace, territoire qui traduisent des conceptions différentes. Les auteurs explicitent peu leur approche du territoire. Tout en affirmant que c'est une notion en évolution constante, les articles n'indiquent pas ou soulignent rarement à quel type de conception du territoire ils font référence. Pourtant trois se dégagent.

La première approche considère le territoire comme donné et clos par des contraintes géographiques, c'est une micro-société au sein de laquelle il y a "le dedans de l'espace régional et le dehors, entre le nous restreint au groupe et les eux du grand large » (Simonin, 1995, 10). Cette dimension du territoire est particulièrement développée dans le premier dossier consacré à un espace régional, l'lle de La Réunion, considérée à la fois comme un la- 
boratoire social et comme un laboratoire des évolutions territoriales par son extériorité insulaire et lointaine (par rapport à la métropole) et par le fait d'être « ce morceau de l'espace national [qui] présente ainsi le privilège d'offrir une situation qui peut éclairer en retour ce qui se passe en métropole et ailleurs ", selon I'expression de Jacky Simonin $(1995,10)$. Cet espace est donc d'abord un lieu, bien circonscrit et sa " configuration socio-spatiale " particulière lui permet d'éclairer des tendances plus larges. Pour le coordinateur du dossier et les auteurs, la société réunionnaise connait « le télescopage de deux espaces sociaux " : il nomme cette société, une "société télescopée " parce qu'elle vit une sociabilité traditionnelle et une sociabilité moderne. Ces deux sociabilités s'expriment de manière conjointe dans les domaines divers : les pratiques télévisuelles, les usages sociaux du téléphone ou de la communication publicitaire. Le dossier appelle à enrichir notre cadre de pensée et à s'engager de manière ambitieuse " dans une activité de déconstruction-recomposition conceptuelle » dont la principale manifestation est la remise en cause d'antagonismes traditionnels entre espace public/espace domestique, société agraire/ société industrielle, centre/périphérie, local/national. Ainsi l'espace local se présente aussi comme un lieu d'observations de tendances qui peuvent être " exportées " à des configurations socio-spatiales autres. C'est au sein de ce dossier que les relations entre l'État et ce territoire sont abordées en termes de violence et de domination. La question de la domination n'a pas été reprise par la suite, mais c'est évidemment une des composantes de l'analyse. Une des explications réside sans doute dans les politiques de communication des collectivités territoriales, centrées en grande partie sur la mise en avant de leur légitimité, de la reconnaissance de leur pouvoir, de la construction de référents identitaires locaux et donc du consensus. Les lectures des phénomènes info-communicationnels en terme de violence et de domination sont, finalement, peu importantes sauf lorsqu'est en jeu les débats, les conflits et les controverses de la sphère publique ( $n^{\circ} 47$ et 48$)$.

La deuxième approche met l'accent sur "l'espace pratiqué », selon l'expression de Michel de Certeau. L'analyse du territoire se réalise à partir de ceux et celles qui le pratiquent. Le dossier « espace urbains, espaces publics, paroles et interprétations des habitants » (EDC n³1), propose ainsi « l'analyse des dispositifs qui permettent la production, l'expression, la constitution des mémoires, des imaginaires, des interprétations, des habitants ${ }^{1}$ de la ville » (Da Lage et al., 2008, 7). Les chercheurs mettent en évidence la place de l'habitant dans les activités info-communicationnelles et elle parait ainsi plus importante que celle de citoyen, d'usager, de militant ou même de public.

Le recours au terme d'habitant met l'accent sur la localisation et plus nettement sur le lien qui est tissé entre un individu et son environnement social. Le qualificatif est souvent présent dans les politiques publiques ou dans les 
discours des responsables politiques locaux, le terme indique la construction d'un rapport avec le territoire. Il est ainsi analysé comme édifié par les politiques de communication des collectivités territoriales ou par les documents produits dans les opérations de concertation, bref les responsables s'adressent à lui, comme à un public particulier, pris sous l'ange de ses attaches locales. C'est à travers plusieurs activités dont la patrimonialisation et la concertation qu'est construite cette figure. Michèle Gellereau, dans le numéro consacré au développement des territoires (EDC $\left.n^{\circ} 26\right)$, met en évidence les objectifs des politiques de patrimonialisation "soit restituer aux habitants un territoire en les éduquant à la lecture des changements [...], soit il s'agit de mettre en scène les habitants eux-mêmes " (Gellereau, 2003, 63). Les expériences de création culturelle font appel à la mémoire, à l'espace vécu et à la "subjectivité du territoire »(Gellereau, 2003, 64). Ainsi la création culturelle et la participation des habitants dans les actions de transmission du patrimoine font partie de la formation des identités territoriales et du lien entre habitants et territoires. Dans le cadre des opérations de concertation, la construction de la figure de I'habitant révèle son ambiguïté et souligne un usage stratégique de l'expression. Jacques Noyer et Bruno Raoul mettent ainsi l'accent sur les contradictions des politiques menées en matière de concertation locale car " l'individu-destinataire de ces opérations est construit, c'est-à-dire 'figuré' et 'projeté' dans les actes et par les discours qui le prennent en compte, 'segmenté' et organisé en figures autonomes (ou non) selon les phases de l'opération - quand bien même, et paradoxalement d'une certaine manière, la parole des habitants se révèle peu convoquée dans les documents " (Noyer et Raoul, 2008, 113). Les conclusions issues de leur étude sur la figure de l'habitant dans les documents de concertation mettent l'accent sur " la 'disciplinarisation' de la fonction habitante " (Noyer et Raoul, 2008, 118). Mais derrière une seule expression, " l'habitant », ou « les habitants", relativement banalisée, plusieurs postures sont identifiées : l'habitant « interlocuteur » des pouvoirs publics, I'habitant imaginé « comme "résident potentiel ", I'habitant comme " partenaire du renouveau », I'habitant protestataire, I'habitant citoyen, habitant " usager-expert des lieux " (Noyer et Raoul, 2008, 124). Traditionnellement, les dénominations utilisées, pour les produits d'information et de communication, font référence aux publics, aux lecteurs et aux spectateurs : avec la figure de l'habitant est proposée une vision désocialisée des acteurs locaux, "désidéologisée », pendant de "l'affaiblissement de l'emploi des catégories de mobilisation telles que 'ouvriers', 'classes populaires', 'classes moyennes', etc., qui faisaient sens » (Anquetin et Freyemuth, 2008 , 26). Paradoxalement, la référence à l'habitant et la construction de ses relations avec le territoire sont mises en évidence dans le cadre d'actions qui font appel à la citoyenneté, c'est-à-dire à la dimension politique des rapports des individus et des collectifs avec la gouvernance. En dépolitisant leur interlocuteur, les pouvoirs publics contribuent à la dépolitisation des enjeux locaux ou plus exactement à l'affaiblissement de l'espace public local. 
La troisième approche envisage, dans une dimension non pas naturelle, ni relationnelle mais dans une dimension institutionnelle, le territoire à travers les représentants politiques, elle est nourrie principalement par les dossiers ayant pour titre " développement des territoires et communication " (EDC $\left.n^{\circ} 26\right)$ et " images de territoires et " travail territorial » des médias (EDC n 37). Il s'agit en premier lieu de la communication des collectivités territoriales, l'analyse met l'accent sur trois dimensions : la mise en avant du territoire, la mise en avant des services publics locaux, la mise en avant de la gestion politique du territoire. Cependant ces caractérisations de la communication des institutions municipales ou départementales ou régionales trouvent leurs développements dans l'évolution des tendances plus profondes : les rapports des individus à leur territoire de vie, leurs rapports à la vie politique, l'évolution de la sociabilité locale. Ainsi les supports d'information et de communication contribuent à ajuster, de manière continue, les structures politiques locales aux transformations et exigences sociales. Dans ce contexte d'ajustement et de réajustement incessant, il est assigné aux techniques d'information et de communication un rôle plus spécifique : celui d'embrigader voire d'enrégimenter les populations dans un type de développement économique et social.

Parmi les territoires étudiés, ce sont les villes qui dominent. Les dossiers consacrés aux « espaces urbains " ( $\left.n^{\circ} 31\right)$ et aux images des territoires $\left(n^{\circ} 37\right)$ sont essentiellement centrées sur les « images de villes » : Villeurbanne, Marseille, Bordeaux, Grenoble. Et lorsque des politiques nationales sont traitées, c'est à travers les politiques de la Ville $\left(n^{\circ} 26\right)$. Ainsi, la territorialisation est-elle observée à travers le prisme des mutations urbaines et de celles-ci uniquement. Et cela se réalise au détriment d'études sur des lieux plus restreints (les quartiers par exemple), ou sur les espaces ruraux ou les « pays » (sauf l'article de M. Briant, $n^{\circ} 26$ ) ou encore sur des espaces particuliers (certains types de banlieues par exemple) ou les « nouvelles Régions ». De même, les « nouveaux territoires " que sont les intercommunalités ne sont pas traités.

Le mouvement de différenciation des territoires s'accompagne d'une intensification des relations entre les différentes institutions. Et la mise en avant de "l'identité territoriale » dans les journaux et autres supports municipaux doit maintenant tenir compte des différentes intercommunalités et de manière plus générale par l'enchevêtrement des différentes collectivités territoriales. Les politiques d'information et de communication se trouvent donc à gérer un double mouvement : celui de la fragmentation des territoires et celui des interrelations entre diverses échelles locales d'une part, celui des pratiques sociales des individus qui relèvent de plusieurs territoires (de vie, de travail, de scolarité, de loisirs, de consommation) d'autre part. Elles jouent ainsi un rôle de rejointoiement entre ces divers territoires, institutionnels, économiques et sociaux. 


\section{2. Médias et territoires}

La place des médias dans les territoires se développe à travers trois axes principaux. Le premier prend en compte les médias locaux eux-mêmes. La presse quotidienne régionale, longtemps média dominant, retient toutes les attentions des chercheurs. Il s'agit de signaler le paradoxe du message journalistique dans la presse régionale, « universel il n'a pas de signification, particulier il est intraduisible en dehors de son contexte " (Breton, 1995, 88). Le deuxième axe considère la presse régionale comme un acteur du territoire. Dans le cadre d'événements culturels, la PQR (presse quotidienne régionale), à travers ses articles, met en valeur la dimension consensuelle de la culture, et il est observé " la co-existence de deux logiques : celle de fédération, de mobilisation d'une population autour d'identités, de symboles, de valeurs communes et celle de performance organisationnelle et publicitaire, la construction d'une image de territoire » (Croissant et Toullec, 2011, 11). La PQR est le centre de l'attention des chercheurs au détriment de l'analyse des autres médias, en particulier la radio mais aussi la presse gratuite, ou encore les télévisions locales. Cette absence de travaux sur les autres médias locaux n'est pas le seul fait de la revue, ce constat pourrait être généralisé à d'autres revues. En outre, la presse locale est peu étudiée dans son organisation (rapports aux lecteurs et aux annonceurs, pratiques journalistiques, pigistes...), elle est surtout mobilisée comme un corpus, e vue de la réalisation d'une analyse de contenu à partir du recueil d'articles. II y a plusieurs raisons à cela. Après une période de développement important au cours des années 70 et 80 , les médias locaux sont intégrés dans des logiques nationales et marchandes qui les absorbent dans des réseaux nationaux (pour les radios locales), ou bien ils demeurent dans une fragilité économique. Leur faible audience et leur spécificité parfois très localisée en font une étude de cas, plus qu'une illustration de tendances plus profondes. Il y a donc peu d'articles sur les médias locaux dans leur activité de production d'articles ou d'émissions, dans la prise en compte de leurs contraintes économiques, peu d'éléments sur l'évolution du marché publicitaire local. De même, la place des professionnels de l'information n'est pas abordée, ainsi que le recrutement des journalistes travaillant dans la PQR, et même leur positionnement dans l'espace politique local. En conséquence, ne sont pas étudiés les problèmes rencontrés par les médias locaux (par exemple la baisse du lectorat), et les nouvelles situations auxquelles ils doivent faire face : la multiplication des autres sources d'informations locales, et la présence des pure players, la professionnalisation du secteur de la communication des entreprises et des services publics.

Les médias sont donc rarement traités pour eux-mêmes et, c'est une des originalités de la revue, ils sont considérés « comme des instances de médiation 
à portée géographique $\aleph^{2}$ (Noyer et Raoul, 2011, 19, constituant ainsi le troisième axe des rapports médias/territoires. À la différence des précédents axes, il ne s'agit pas de prendre en compte la manière dont les médias rendent compte de la vie locale, ni de la manière dont ils contribuent à la fabrique de référents identitaires. L'objectif est de prendre en compte la " matérialité spatiale » et d'en étudier sa mise en forme au sein des médias ou des dispositifs numériques. Le " travail territorial » des médias (et des nouveaux médias) prend en compte le contenu des médias pour analyser les lieux et le sens des lieux qu'ils façonnent. Bref, le travail territorial des médias s'inscrit dans la production de significations par lesquelles «le territoire accède à la reconnaissance » (Bryon Portet, 2011, 30). La notion de reconnaissance, bien que très utilisée dans d'autres domaines, nous semble fructueuse, à plus d'un titre. Elle renvoie aux modalités de visibilité, aux productions de référents (la mémoire des lieux par exemple), et aux constructions de légitimités.

\section{3.}

\section{Les questions territoriales dans la sphère publique}

Le terme « d'espace " dans l'expression « espace public » présente une dimension métaphorique qui tire son origine du titre français de l'ouvrage de Jürgen Habermas. L'espace public au sens de sphère publique politique de débats, d'argumentation et de controverses, est donc à différencier de la notion de lieu ou de territoire. D'ailleurs, l'expression renvoie à deux figures distinctes, celle de citoyen et celle d'habitant. Les deux sens du mot " espace ", souvent utilisés l'un pour l'autre, génèrent des confusions conceptuelles. Mais l'obligation de clarification et de distinction a conduit à opposer « espace symbolique » et " espace physique " plutôt qu'à articuler les deux expressions et à considérer que cette articulation peut être féconde. Cette dimension est prise en compte dans les numéros de la revue mais reste peu éclaircie.

De manière générale, c'est la temporalité de l'espace public qui est prise en compte plus que sa territorialité. Par exemple, dans le cadre des controverses politiques, l'accent est mis sur une périodisation : " on s'intéresse alors à son émergence, sa publicisation, sa dispersion dans plusieurs arènes, ses phases de reflux, de renaissance, éventuellement de clôture »(Rennes, 2016, 27). II est ainsi proposé, à juste titre, de ne pas « écraser ces différents niveaux de temporalité : celle des protagonistes participant et alimentant successivement la controverses et celle, souvent de plus longue durée, des formes argumentatives qu'ils se transmettent sans les avoir eux-mêmes élaborées » (Rennes, 2016, 27). La territorialité de l'espace public politique ou sociétal est peu abordée sans doute pour plusieurs raisons : la première pourrait venir justement de cette 
proximité avec la spatialité contenue dans l'expression elle-même, l'utilisation d'un même terme ne rendant pas flagrante ses différences. La deuxième est liée aux origines de l'expression - l'ouvrage d'Habermas - dans lequel c'est la dimension nationale de l'espace public qui est prise en compte (même si sa localisation, dans des salons, dans des cafés ou dans des lieux comme l'Assemblée nationale est bien signalée), et cette dimension nationale est encore plus fortement présente en France, du fait du caractère centralisateur de l'État. La troisième vient également du fait que le territoire est un opérateur de singularisation, dans un pays dont la tradition est de désingulariser les lieux et « d'aménager les territoires ».

L'on pourrait ainsi considérer que l'espace public s'est développé contre le territoire. Mais ce rapport a évolué et les différents dossiers de la revue - et plus particulièrement les numéros 47 et 48 - mettent en évidence deux types de relations entre espace public et territoires, avec des intensités différentes : le territoire comme un enjeu de l'espace public, le territoire comme un modèle de l'espace public.

La première, la plus fréquemment présente, renvoie au fait que les transformations des territoires soulèvent des oppositions : un nouveau quartier, un nouvel équipement urbain, mais aussi des projets d'aménagement routier, portuaire ou aéroportuaire, ou d'installation de centres de loisirs par exemple. C'est le cas des luttes menées contre le projet d'aéroport de Notre-Dame-des-Landes et plus généralement contre les projets de transformation des territoires, projets qui trouvent une justification dans le développement économique du territoire.

Les projets donnent lieu à des conflits qui rejettent, ici comme dans d'autres domaines, les dispositifs de participation et de concertation institutionnalisées comme le sont les enquêtes publiques ou bien encore les débats organisés dans le cadre de la CNDP (Commission Nationale du Débat Public). Dans ces situations, et pour les opposants au projet, les médias « sont perçus comme étant au service du 'système' et incapables de prendre en compte leurs visions du monde et leurs arguments " (Mabi, 2016, 113) Le travail des journalistes se trouve disqualifié car présenté comme étant au service de "l'espace public dominant ", selon l'expression de Peter Dahlgren (2000). Cette mise en doute systématique des structures de concertation et des médias conduit les militants à développer leurs propres stratégies de médiatisation. Se retrouve dans cette utilisation des réseaux sociaux numériques une tradition des mouvements sociaux pour produire une information qui se veut alternative aux médias. Ainsi la presse de " contre-information ", la vidéo militante, les radios locales, ont été créées et développées pour proposer et imposer une autre « problématisation " d'une situation sociale. L'utilisation des réseaux sociaux numériques s'inscrit dans cette histoire de mobilisation des médias et nouveaux médias et a pour objectif de « rendre visible ses arguments et influencer les publics dans la phase de construction des problèmes. Ce temps de problématisation est un enjeu politique déterminant si l'on considère l'espace public comme 
un lieu de formation du jugement des publics » (Mabi, 2016, 127). La " mobilisation » des dispositifs numériques et leur utilisation différenciée souligne la professionnalisation des opposants dans ce domaine, elle indique également leur capacité à « nationaliser » un enjeu local.

La seconde dimension porte sur le territoire comme un modèle alternatif aux modèles dominants de gestion des affaires publiques et comme modalité d'action et d'engagement des individus. C'est localement que les engagements pour des causes nationales se développent, c'est localement que les pratiques militantes s'organisent. La proximité territoriale constitue ainsi un ressort important de la formation " politique " des individus et les pratiques locales peuvent dessiner des modèles innovants. C'est le cas d'une cantine autogérée et des assemblées générales qui possèdent quelques points communs avec la conception classique de l'espace public que propose Habermas. "À l'instar des bourgeois chez Habermas, les militants sont ici des personnes privées qui se constituent en public pour discuter de leurs intérêts en faisant usage de la raison et en étant animés par la recherche du consensus " (Robineau, 2016, 142). La cuisine et ses modalités de fonctionnement qui ont pour objectif « de sortir de l'entre soi militant par un renouvellement des pratiques militantes " (Robineau, 2016, 134) offrent des situations d'expérimentations. Les militants demeurent très vigilants vis-à-vis des médias, en refusant les rapports avec les grands médias généralistes pouvant " présenter des risques de dépossession et de transformation d'une parole collective " (Robineau, 2016, 136), l'utilisation des réseaux sociaux numériques est également remise en cause. Et la presse militante exige des processus de vérification, d'approbation et de "labellisation » longs et exigeants. Bref c'est le lieu même de la cuisine qui favorise un espace public en action, " cet espace public local est avant tout un espace public utilisé à la fois comme lieu de débat, d'organisation et d'action politique " (Robineau, 2016, 144) pour des militants qui s'adressent avant tout à des publics locaux et non à des institutions étatiques.

Entre ces deux configurations, le territoire comme enjeu de l'espace public et le territoire comme modèle alternatif à l'espace public, les situations analysées dans les différents dossiers de la revue sont fort diverses : elles couvrent les conditions d'émergence d'un espace public (Watin et Wolff, 1995), les dimensions procédurales de l'espace public dans les opérations de concertation (Noyer et Raoul, 2008), et les « ressorts de la conflictualité dans les espaces publics contemporains »(Dalibert et Quemener, 2017).

II est pourtant une dernière dimension qui est peu analysée, c'est celle de la place de l'État dans la territorialisation de l'espace public. Dans le cadre des luttes contre le sida, par exemple, les associations se sont trouvées enrôler. En effet, la communication publique accompagne l'action de l'État dans un processus d'institutionnalisation de normes liées à la prévention contre le sida. Elle conduit à une " intégration des acteurs associatifs et de leurs discours concurrents au sein de modalités consensuelles de co-construction de 
l'action publique, nécessaires à l'action publique comme à son efficacité » (De Oliveira, 2017, p. 83). Dans ce cadre, il y a bien affrontement entre différents acteurs et l'enjeu de l'affrontement est bien l'imposition de normes sociales et leur contrôle.

La question territoriale est enrichie traditionnellement par des études monographiques. L'une des difficultés principes du chercheur est alors de replacer son analyse, souvent très circonscrite à un espace bien délimité dans des grandes tendances : la fragmentation de plus en plus fine de l'espace public (Gadras et Pailliart, 2013, 24) ? La polarisation et la spécialisation des activités sociales et économiques ? L'enrôlement des individus, au plus près des pratiques sociales, dans des modèles de développement ? Finalement, la question territoriale c'est-à-dire l'analyse des processus de territorialisation reflète bien les exigences des chercheur.e.s en sciences humaines et sociales: la gestion de la courte et de la longue durée, la connaissance intime d'un lieu et la maitrise de son dépassement et de son élargissement. 
Bibliographie

Anquetin V., Freyermuth A. (2008). La figure de "I'habitant ", Rennes, Presses Universitaires de Rennes.

Breton P. (1995). " La presse régionale entre le fait universel et le commentaire local ». In Études de communication, $n^{\circ} 17$, p. 73-89.

Bryon Portet C. (2011). « Les productions télévisées, genre oublié dans la construction de l'image d'un territoire? L'exemple de co-construction de l'image socioculturelle de la ville de Marseille par la série Plus belle la vie ». In Études de communication, $n^{\circ} 37$, p. 79-96.

Croissant V., Toullec B. (2011). « De la coopétition des territoires au consensus médiatique. L'exemple du traitement médiatique d'événements culturels par la presse régionale ». In Études de communication, $n^{\circ} 37$, p. 97-114.

Da Lage É., Gellereau M., Laudati P. (2008). «Introduction ». In Études de communication, $\mathrm{n}^{\circ}$ 31, p. 7-14.

Dahlgren P. (2000). « L'espace public et l'internet. Structure, espace et communication ". In Réseaux, n¹00, p. 157-186.

Dalibert M., Quemener N. (2017). «Introduction ». In Études de communication, $\mathrm{n}^{\circ} 48$, p. 7-20.

De Oliveira J.-P. (2017). « La communication publique comme support de légitimation et d'institutionnalisation des normes sociales : le cas de la prévention du sida ». In Études de communication, $n^{\circ} 48$, p. 71-90.

Gadras S., Pailliart I. (2013). " Les territoires et les médias dans la construction de l'espace public ». In Noyer J., Raoul B., Pailliart I. (dir.), Médias et territoires. L'espace public entre communication et imaginaire territorial, Villeneuve d'Ascq, Presses Universitaires du Septentrion.

Grémion P. (1996). Le pouvoir périphérique. Bureaucrates et notables dans le système politique français, Paris, Le Seuil.

Kada N., Pasquier R., Courtecuisse C., Aubelle V. (2017). Dictionnaire encyclopédique de la décentralisation, Boulogne Billancourt, Berger Levrault.

Mabi C. (2016). « Luttes sociales et environnementales à l'épreuve du numérique : radicalité politique et circulation des discours ». In Études de communication, $n^{\circ} 47$, p. 111-130.

Mabileau A. (1985). « Les institutions locales et la relation centre-périphérie ». In Grawitz M. et Leca J. (dir.), Traité de science politique, vol. 2, Paris, PUF, p. 553-598.

Meyriat J., Miège B. (2002). « Le projet des SIC : de l'émergent à l'irréversible ». In Boure R. (dir.), Les origines des sciences de l'information et de la communication. Regards croisés, Villeneuve d'Ascq, Presses Universitaires du Septentrion, p. 45-70. 
Moeglin P. (1991). " Télématique : de la recherche sur les usages aux usages de la recherche ". In Bulletin du CERTEIC, $\mathrm{n}^{\circ} 12$, p. 23-50.

Noyer J., Raoul B. (2008). "Concertation et 'figures de I'habitant' dans le discours des projets de renouvellement urbain ». In Études de communication, $\mathrm{n}^{\circ} 31$, p. 111-130.

Noyer J., Raoul B. (2011). «Le 'travail territorial' des médias. Pour une approche conceptuelle et programmatique d'une notion ". In Études de communication, $\mathrm{n}^{\circ} 37$, p. 15-46.

Pailliart I. (2003). « Une histoire des formes communicationnelles de la démocratie locale ». In Sciences de la société, $n^{\circ} 60$, p. 30-46.

Rennes J. (2016). « Les controverses politiques et leurs frontières ». In Études de communication, $n^{\circ} 47$, p. 21-48.

Robineau C. (2016). « Constituer un contre-public en marge des médias : négociations, circulations et normativités d'un discours 'révolutionnaire' au sein d'une cantine de quartier ». In Études de communication, $n^{\circ} 47$, p. 131-148.

SFSIC (dir.) (1984). Actes du congrès Inforcom 1982, "Information, économie et société », Grenoble, Presses Universitaires de Grenoble et SFSIC. de communication, $n^{\circ} 17$, p. 7-11.

Watin M., Wolff E. (1995).

"L'émergence de l'espace public à La Réunion. Un contexte socio-historique singulier ». In Études de communication, $\mathrm{n}^{\circ} 17$, p. 19-39.

\section{Références Études de communication}

Études de communication, Dossier Médias et communication dans un espace régional, n 17, 1995.

Études de communication, Dossier Développement des territoires et communication : politiques et pratiques à l'œuvre, n² 26, 2003.

Études de communication, Dossier Espaces urbains, Espaces publics, Paroles et interprétations des habitants, $n^{\circ} 31,2008$.

Études de communication, Dossier Images de territoires et " travail territorial » des médias, n 37, 2011.

Études de communication, Dossier Circulation et qualification des discours. Conflictualités dans les espaces publics (1), n 47, 2016.

Études de communication, Dossier Rapports sociaux et hégémonie. Conflictualités dans les espaces publics (2), n 48, 2017.

Simonin J. (1995). «Présentation. Les médias et la formation d'un espace public régional ». In Études 